\title{
"Tip-In" or "Randezvouz" techniques as a first choice in retrograde chronic total occlusion interventions
}

\section{-Mihajlo Kovačić*, DDario Dilber}

County Hospital Čakovec, Čakovec, Croatia
RECEIVED:

February 9, 2020

ACCEPTED:

February 22, 2020

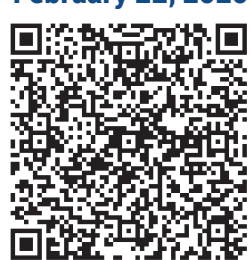

KEYWORDS: percutaneous coronary intervention, chronic total occlusion, retrograde, tip-in, rendezvous CITATION: Cardiol Croat. 2020;15(3-4):49-50. | https://doi.org/10.15836/ccar2020.49

*ADDRESS FOR CORRESPONDENCE: Mihajlo Kovačić, Županijska bolnica Čakovec, I.G.Kovačića 1E, HR-40000 Čakovec, Croatia. / Phone: +385-98-266-774 / E-mail: mihajlo1983@gmail.com

ORCID: Mihajlo Kovačić, https://orcid.org/0000-0002-2577-9474 • Dario Dilber, https://orcid.org/0000-0002-0062-4708

IIIIIIIIIIIIIIIIIIIIIIIIIIIIIIIIIIIIIIIIIIIIIIIIIIIIIIIIIIIIIIIIIIIIIIIIIIIIIIIIIIIIIIIIIIIIIIIIIIIIIIIIIIIII

Introduction: The "Tip-In" method is a technique first described in 2015 and is developed to overcome some limitations of commonly used methods in retrograde approach in chronic total occlusion (CTO) percutaneous coronary intervention (PCI).1,2 The most commonly applied retrograde technique is placing a guidewire just distal to the distal cap using collateral vessels, with subsequent retrograde crossing of the occlusive segment which is followed by advancement of a microcatheter and externalization of a long guidewire to allow PCI, but sometimes the microcatheter fails to advance into the antegrade guide, obstructing attempts at guidewire externalization. ${ }^{3}$ The "tip-in" technique involves the advancement of an antegrade microcatheter over the retrograde guidewire enabling subsequent antegrade wiring of the CTO segment and facilitates successful completion of a retrograde CTO procedure. Wiring can be completed by threading an antegrade wire through the retrograde microcatheter and crossing the CTO which is named rendezvous method. To our knowledge there are no published reports of a use of this technique as a primary strategy in retrograde CTO. Here we present a case where the "tip-in" technique was used not as a "bail-out" method after an unsuccessful completion of an applied retrograde technique, but as a first line strategy of retrograde approach.

Case report: 57-year-old male patient was presented with angina class III. Echocardiography shoved good ejection fraction of the left ventricle with viable myocardium. Coronary angiography showed occlusion of the mid-segment of the right coronary artery (RCA) with J-CTO 3 score (ambiguous cap, length, calcium). With bilateral vascular access, left snuffbox and right transulnar, Amplatz left 0.75 $7 \mathrm{~F}$, and Extra backup 3.5 6F guiding catheters were engaged to the right and left coronary arteries respectively. Several methods were tried in an anterograde manner with the support of Corsair Pro 135 microcatheter (Asahi Intecc). First, anterograde wire escalation (AWE) technique, with wires Sion, Gaia first, Gaia second, Gaia third, was attempted with many redirections, always subintimal. (Figure 1A) Second, the parallel wire technique was tried unsuccessfully (Figure 1B), and also anterograde fenestration technique (AFR) with Sion Black wire, and 2.5x15 SC balloon on Gaia third was unsuccessful, due to probably too small balloon (Figure 1C). The bigger balloon was planned, but with retrograde injection, the subintimal hematoma was compressing the distal vessel. Switch to the retrograde procedure was done with Corsair Pro 150 microcatheter and septal surfing with Sion and finally Suoh03 wire through long septo-epicardial collateral (Figure 1D). Retrograde wire escalation (RWE) was not succesfull after that reverse CART method was used to externalize wire towards the anterograde guiding catheter (Figure 1E). Rather than the externalization wire technique, the "tip-in" maneuver was done with retrograde wire positioning into anterograde microcatheter on the convex part of the anterograde guiding catheter (Figure $\mathbf{~} \mathbf{F}$ ). After that, anterograde microcatheter was advanced over the retrograde guidewire distal to the CTO, to convert the procedure to the anterograde method. Finally, a good result was achieved with the implantation of 3 drug-eluting stents. (Figure 1G)

Discussion: Tip-in or rendezvous techniques with fast conversion to the anterograde procedure are suggested as a first approach because of some pitfalls of classic externalization wire technique. First, there is no need for long externalization wires like RG3 from Asahi Intecc, or R350 from Teleflex. Second, in many cases of the retrograde procedure, there are difficulties in microcatheter crossing over the collaterals, bandings and calcified lesions or due to a shortage of microcatheter length. Third, excessive rotation and pushing of the microcatheter increase the risk of vessel injury. Fourth, prolonged duration of retrograde gear position is producing donor vessel ischemia, which can lead to left system injury, like left main trauma, etc. Fifth, with retrograde equipment in situ, there is a need for more meticulously activated clotting time (ACT) controlling, with much higher ACT needed, which can result in more bleeding. Sixth, in some cases, especially when snaring of the wire is performed, the spring coil segment of the externalized wire is disrupted, which can compromise navigation of the materi- 
"Tip-In" or "Randezvouz" techniques as a first choice in retrograde chronic total occlusion interventions

als to the wire. Seventh, the externalized wire can be stuck in the system, and extraction can produce serious complications like left main and ostial right coronary artery lesion, collaterals trauma/dissection, "cutting" of the interventricular septum, and some examples of so-called „chocking heart" phenomenon. Accordingly, tip-in and rendezvous methods cannon provide strong support in delivering balloons and stents as the externalization method can, which is the main advantage of this technique. Another main pitfall of tip-in and rendezvous is that one can easily lose anterograde wire position and with that putting the whole procedure to the beginning.

Conclusion: In this case report, we demonstrated that the "tip-in" technique can be successfully obtained as a first-line strategy in retrograde CTO with lower cost and rate of possible complications of the procedure as this technique doesn't require an "externalization" wire, with fast extraction of retrograde equipment and movement towards the straightforward anterograde procedure.
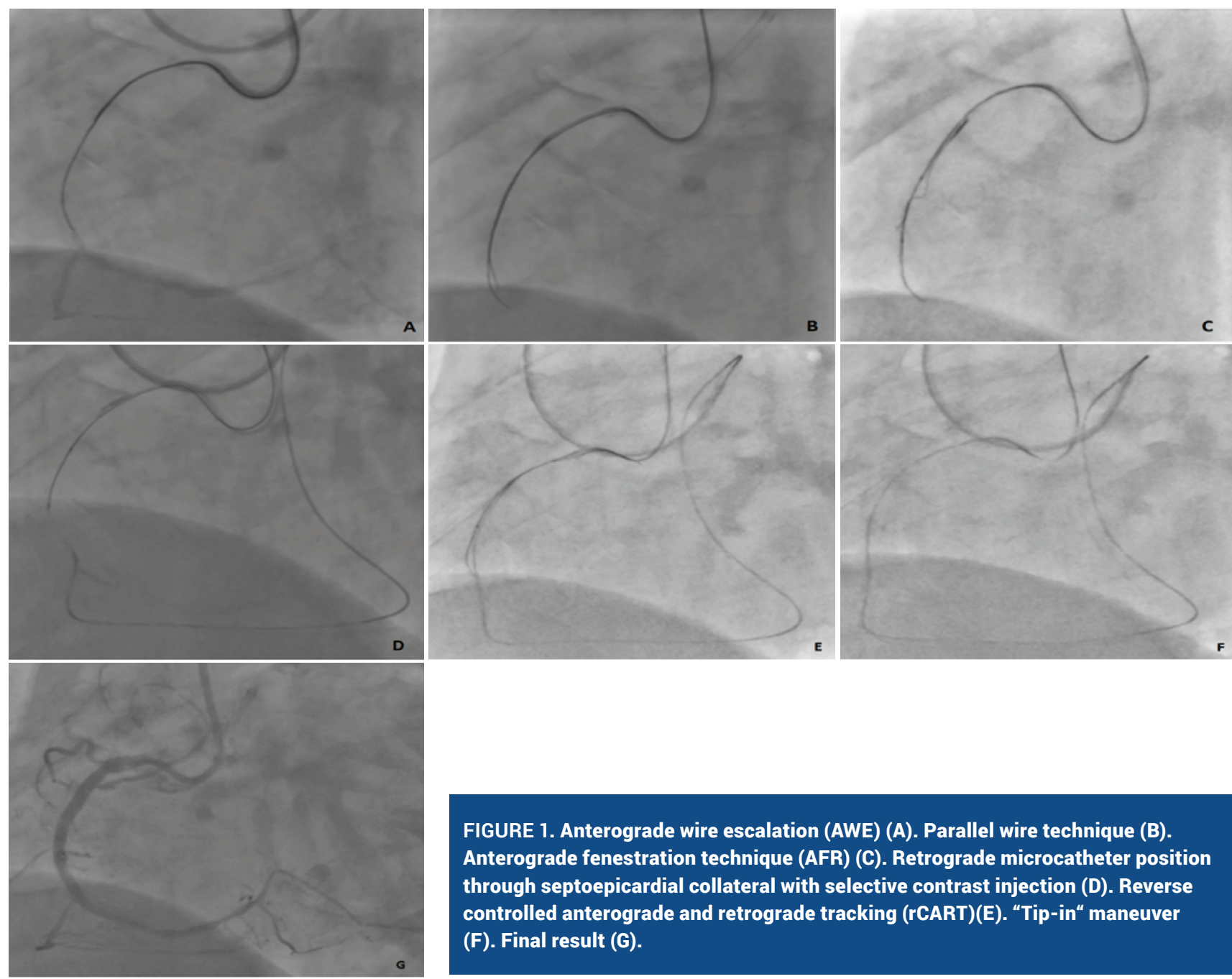

FIGURE 1. Anterograde wire escalation (AWE) (A). Parallel wire technique (B). Anterograde fenestration technique (AFR) (C). Retrograde microcatheter position through septoepicardial collateral with selective contrast injection (D). Reverse controlled anterograde and retrograde tracking (rCART)(E). "Tip-in" maneuver (F). Final result (G).

LITERATURE IIIIIIIIIIIIIIIIIIIIIIIIIIIIIIIIIIIIIIIIIIIIIIIIIIIIIIIIIIIIIIIIIIIIIIIIIIIIIIIIIIIIIIIIIIIIIIIIIIIIIIIIIIIIIIIIIIIIIIIIIIIIIIIIIIIIIIIII

1. Vo MN, Ravandi A, Brilakis ES. "Tip-in" technique for retrograde chronic total occlusion revascularization. J Invasive Cardiol. 2015 May;27(5):E62-4. PubMed: https://www.ncbi.nIm.nih.gov/pubmed/25929304

2. Yamane M. "Tip-In" method: a novel, wire randez-vous method in need of retrograde CTO PCI. Srce i krvni sudovi. 2015; 34(1):9-13. https://doi.org/10.5937/siks1501009Y

3. Mishra S. Unraveling the mystique of CTO Interventions: Tips and techniques of using hardware to achieve success. Indian Heart J. 2017 Mar - Apr;69(2):266-276. https://doi.org/10.1016/j.ihj.2017.02.004 